\title{
The safety and effectiveness of volumetric magnetic resonance-guided high-intensity focused ultrasound treatment of symptomatic uterine fibroids: early clinical experience in China
}

Rui Chen ${ }^{3}$, Bilgin Keserci ${ }^{2}$, Hui Bi ${ }^{3}$, Xiaobing Han ${ }^{4}$, Xiaoying Wang ${ }^{5}$, Wenpei Bai ${ }^{3}$, Yueling Wang ${ }^{4}$, Xuedong Yang ${ }^{5}$, Jian Yang ${ }^{6}$, Juan $\mathrm{Wei}^{7}$, Minna Seppälä ${ }^{8}$, Antti Viitala ${ }^{8}$ and Qinping Liao ${ }^{1,3^{*}}$

\begin{abstract}
Background: Uterine fibroids are the most common benign tumor in women, and surgical intervention is still the main fibroid treatment. Patient demands have encouraged development of less-invasive methods such as high-intensity focused ultrasound (HIFU). This study aimed to evaluate the safety and effectiveness of magnetic resonance-guided high-intensity focused ultrasound therapy using a volumetric ablation technique in the treatment of symptomatic uterine fibroids in China.

Methods: One hundred and seven patients were enrolled and treated with magnetic resonance-guided highintensity focused ultrasound in this study. Clinical efficacy was based on the proportion of patients with fibroid shrinkage (10\% volume reduction or more compared to baseline) at 6 months post treatment as measured with magnetic resonance imaging. The quality of life and symptom outcome was assessed using the uterine fibroid symptom and quality of life questionnaire with symptom severity scoring. Safety was primarily assessed by evaluating the reported adverse events.

Results: Ninety nine of the 107 treated patients had fibroid shrinkage at 6 months post treatment. Resulting in an overall $93 \%$ (95\% confidence interval $86-97 \%$ ) treatment success rate, $p$ value $<0.001$; the symptom severity scoring and health-related quality of life at 6 months was statistically different from the screening symptom severity scoring at 0.05 level. Of 366 adverse events reported, there were no study procedure-related or device-related serious adverse events were in the study.
\end{abstract}

Conclusions: This study demonstrated that the volumetric magnetic resonance-guided high-intensity focused ultrasound device is safe and technically effective and can be utilized in clinically efficient treatments of symptomatic uterine fibroids.

Trial registration: NCT01588899

Keywords: MR-guided high-intensity focused ultrasound, Therapy outcome, Uterine fibroids, Volumetric ablation technique

\footnotetext{
* Correspondence: 13701124527@163.com

'Department of Obstetrics \& Gynecology, Beijing Tsinghua Changgung

Hospital, Medical Center, Tsinghua University, No. 168 Litang Road,

Changping District, Beijing, China

${ }^{3}$ Department of Obstetrics \& Gynecology, Peking University First Hospital,

Beijing, China

Full list of author information is available at the end of the article
}

\section{) Biomed Central}

(c) 2016 The Author(s). Open Access This article is distributed under the terms of the Creative Commons Attribution 4.0 International License (http://creativecommons.org/licenses/by/4.0/), which permits unrestricted use, distribution, and reproduction in any medium, provided you give appropriate credit to the original author(s) and the source, provide a link to the Creative Commons license, and indicate if changes were made. The Creative Commons Public Domain Dedication waiver (http://creativecommons.org/publicdomain/zero/1.0/) applies to the data made available in this article, unless otherwise stated. 


\section{Background}

Uterine fibroids are the most common benign tumor in pre- and peri-menopausal women. As fibroids increase in size, they can produce pain, menorrhagia, fertility problems, pressure, bloating, and urinary and bowel compression symptoms [1-4].

Treatment options for symptomatic uterine fibroids include surgery (myomectomy or hysterectomy), drug treatment (gonadotropin-releasing hormone analogs) [5], minimally invasive techniques such as uterine artery embolization (UAE) [6-8], and cryotherapy [9, 10]. Even though surgical interventions of uterine fibroids have been the most common treatment option, it requires anesthesia, hospital stays, and long recovery periods. It has been estimated that 600,000 hysterectomies are performed per year in the USA and more than half of the conducted hysterectomies are due to fibroids [11, 12]. Recently, patient demands have encouraged development of lessinvasive methods such as UAE, ultrasound-guided highintensity focused ultrasound (US-HIFU) and magnetic resonance-guided high-intensity focused ultrasound (MRHIFU).

In HIFU treatment, the beam of HIFU penetrates through soft tissue creating localized high temperatures (55 to $70{ }^{\circ} \mathrm{C}$ ) for a few seconds within the target, thus producing irreversible cell damage and coagulative necrosis. Applying HIFU energy to a fibroid tissue requires treatment planning, targeting of the US beam to the desired locations and monitoring of the energy delivery. In some applications, this can be performed using diagnostic US imaging in combination with the HIFU. While diagnostic US provides some anatomical details, helps with treatment planning and targeting, and has a strength in terms of motion, for instance, fibroid motion caused by respiration or motion of adjacent bowel that can cause artifact in MR imaging, it does not provide $3 \mathrm{D}$ planning or means of measuring the temperature increase. MR imaging provides a non-invasive temperature measurement, thermal dose quantification in the target tissue, utilizing the protonresonance frequency (PRF) shift phenomena to monitor the temperature [13], and continuous imaging of the fibroid and surrounding structures such myometrium, bowel, and sacral nerves.

Previous studies have shown that MR-HIFU is capable of reducing the fibroid size and fibroid-related symptoms while maintaining an excellent safety profile [14-29]. In these studies, ablation of fibroid volumes is either done by the conventional approach, point-by-point technique, or by a volumetric heating technique. The first one is performed by iterative sonication of a single focal point, with each sonication followed by a cooling period. However, with this approach, a relatively large portion of the delivered energy is lost via diffusion of heat out of the small targeted region, and long treatment times are required.
On the other hand, a volumetric heating technique where the focus of the ultrasound beam is electronically steered along a trajectory comprising of multiple outward-moving concentric circles with an axial diameter of 4-16 $\mathrm{mm}$ has been introduced [22, 30, 31].

The aim of this study was to evaluate the safety and effectiveness of MR-HIFU therapy using a volumetric ablation technique in the treatment of symptomatic uterine fibroids in China.

\section{Methods}

This study was a multi-center, single arm, non-randomized clinical trial to evaluate the safety and effectiveness of volumetric MR-HIFU system in the treatment of symptomatic uterine fibroid patients (sponsored by Philips, clinicaltrials.gov identifier NCT01588899). Local ethics committee approval was obtained from both Peking University First Hospital and the First Affiliated Hospital, Xian Jiaotong University for the protocol prior to study initiation. Written informed consent was obtained from each patient at the screening visit prior to the initiation of any study-related procedures.

A total of 350 patients were screened for the trial, out of which 107 were included. Inclusion criteria were as follows: (1) women aged >18 years; (2) weight <140 kg; (3) pre- or peri-menopausal; (4) MR-HIFU device accessibility to treat at least $50 \%$ of the total fibroid volume; (5) total planned ablation volume of all fibroids should not exceed $250 \mathrm{ml}$; (6) dominant fibroid (diameter) is greater than or equal to $3 \mathrm{~cm}$; (7) no highly perfused or brighter than myometrium in T2-weighted MRI (a.k.a. type 3 per Funaki classification [32]); and (8) willing and able to attend all study visits. Exclusion criteria were as follows: (1) other pelvic disease; (2) desire for future pregnancy; (3) positive pregnancy test; (4) hematocrit $<25 \%$; (5) surgical clips in the direct path of the HIFU beam; and (6) MRI contrast agent contraindicated.

All therapies were conducted using a clinical MR-HIFU system (Sonalleve V2, Philips, Best, the Netherlands) integrated into a 1.5T MR scanner at Xi'an site and 3.0T MR at Peking site (Achieva, Philips, Best, the Netherlands). The details of the MR-HIFU system and treatment procedures have been described elsewhere [16]. T2-weighted (T2W) 3D turbo spin-echo (TSE) images were acquired for screening and treatment planning. RF-spoiled gradient-recalled EPI sequence used for real-time thermometry had three coronal slices perpendicular to the beam axis, centered at the focal-region and one sagittal slice aligned along the beam propagation direction and two additional slices were positioned to monitor potential excessive near field (i.e., on the skin) and far-field (Fig. 1a-d). Immediately after HIFU treatment and at follow-ups, a contrast-enhanced T1-weighted (CE-T1W) image was acquired. 


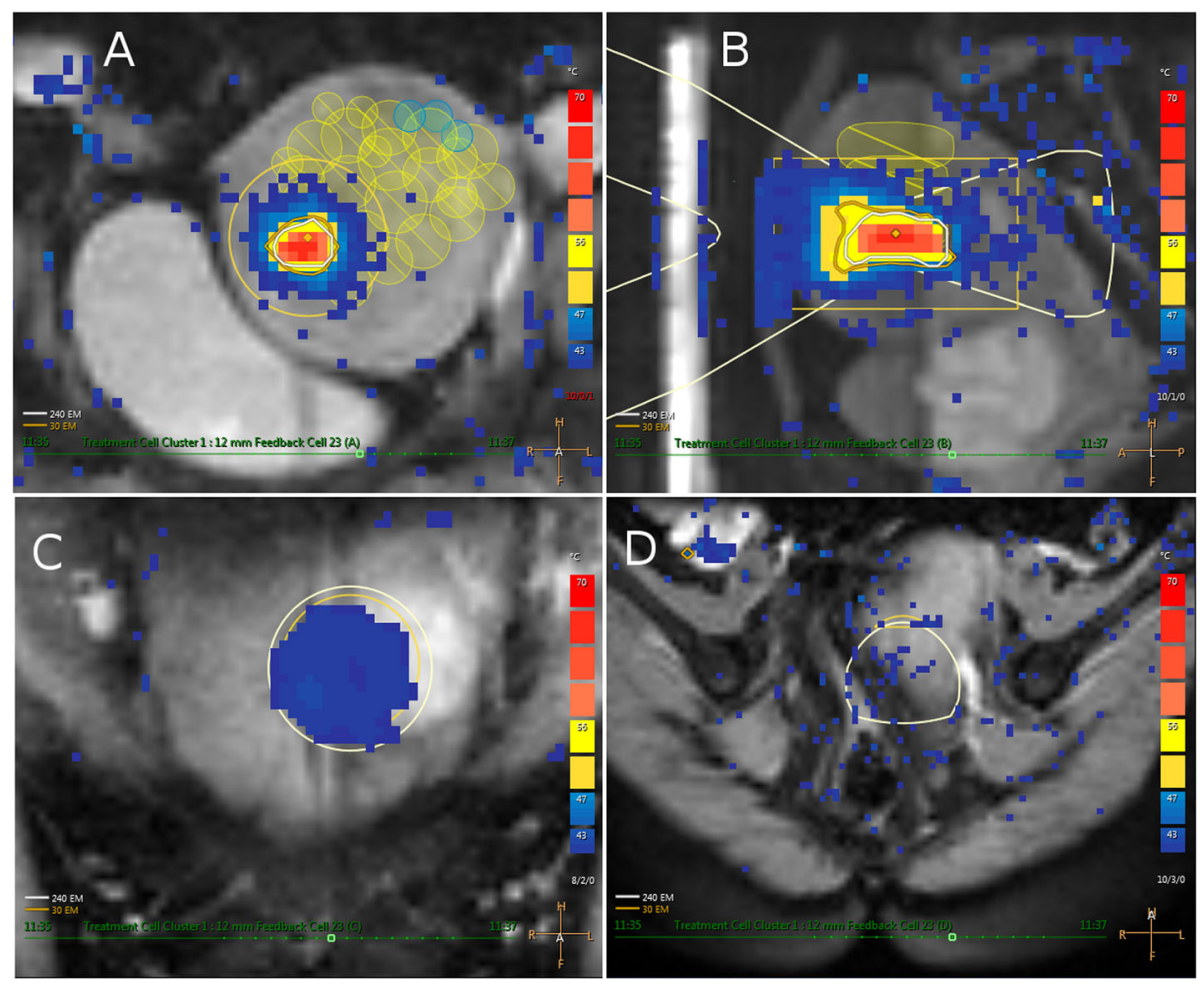

Fig. 1 Multiplane MR thermometric images acquired during sonication with a 12-mm treatment cell (frequency, 1.2 MHz; acoustic power, $190 \mathrm{~W}$ ) with visualization of the ultrasound focus in $\mathbf{a}$ coronal view, $\mathbf{b}$ sagittal view, and $\mathbf{c}$ coronal view from the near field and $\mathbf{d}$ oblique view from the far field

Patient immobilization time was limited to $3 \mathrm{~h}$ due to the risk of deep venous thrombosis. For analgesics, all patients received $200 \mathrm{mg}$ Celebrex at Peking site and oxycodone hydrochloride and Paracetamol (acetaminophen) tablets (5 $\mathrm{mg})$ at Xi'an site.

\section{Study endpoints}

\section{The primary endpoints}

The primary endpoint of the study, clinical efficacy, was based on the proportion of treated patients with fibroid shrinkage as measured with MRI without additional treatment (myomectormy, hysterectomy, uterine artery embolization, or MR-HIFU) during the follow-up period $[20,25,26]$. An image-based primary endpoint was selected in order to be in line with the Chinese SFDA Notice 222 (2007): Notice on the issuance of the relevant technical requirements for HIFU device.

Women were defined as having fibroid shrinkage, i.e., treatment success at 6 months if the total volume of their treated fibroids plus measurement error estimate was $90 \%$ or less then than the baseline fibroid volume minus measurement error estimate. Similarly, women were considered as treatment failures (no fibroid shrinkage) if the total volume of their treated fibroids at 6 months plus measurement error estimate was more than $90 \%$ of the total baseline fibroid volume minus measurement error.
The estimation of the measurement error was based on intra-reader variability and was calculated by the core laboratory responsible for image evaluation. Fibroid volume was estimated by manual segmentation on T2W MRI at baseline (treatment day, prior to treatment) and at 1,3 , and 6 months post treatment. Measurements were performed by an independent reader (CRO's core lab) blinded to the patient information. All patients who underwent HIFU treatment were to be included in the primary efficacy analysis.

In addition to the primary endpoint expressed as a binary variable, the percent reduction in fibroid volume was also calculated using the following formula:

$$
\% \text { Reduction }=\frac{\text { Total Volume at Baseline }- \text { Total Volume at } 6 \text { months }}{\text { Total Volume at Baseline }} \times 100
$$

\section{The secondary endpoints}

In this study, the secondary endpoints were chosen to match endpoints commonly used internationally in the field of fibroid follow-up.

- Evaluation of the non-perfused volume assessed by MRI: The non-perfused volume (NPV) was estimated using a manual contour segmentation on CE-T1W images immediately post treatment, 1,3 , and 6 months 


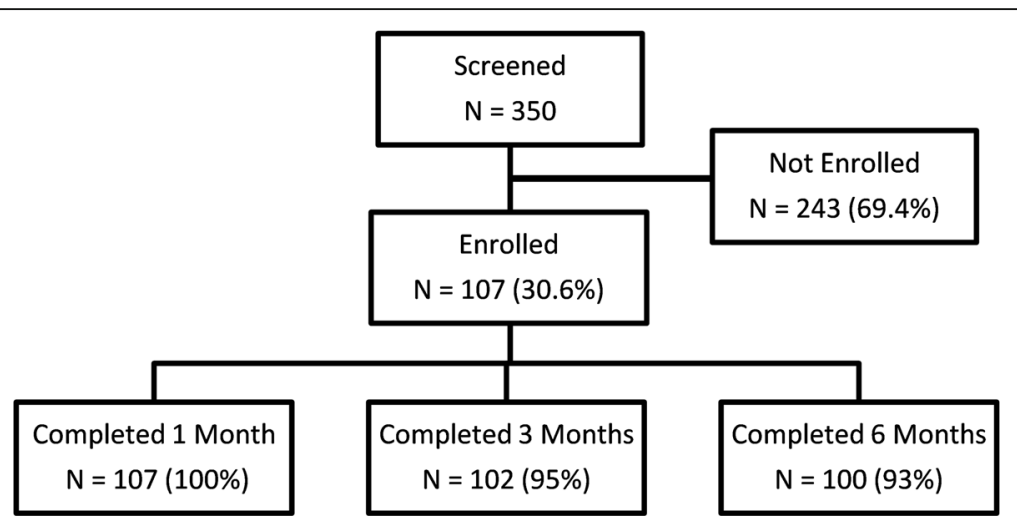

Fig. 2 Flowchart of patient screening and enrollment

post treatment. Lesion NPV measurements were performed by an independent reader (CRO's core lab) blinded to the patient information. The percentage of the fibroid volume that was non-perfused was calculated as follows:

$\operatorname{NPV}(\%)=\frac{\text { Non }- \text { perfused Volume }}{\text { Fibroid Volume }} \times 100$

- Evaluation of The Uterine Fibroid Symptom and Quality of Life (UFS-QoL) questionnaire with symptom severity scoring (SSS): UFS-QoL questionnaire was completed at screening, 1, 3, and 6 months post treatment. The UFS-QoL has seven subscales (i.e., SSS, concern, activities, energy/mood, control, selfconscious, and sexual function) and was validated in a study comparing scores between women with normal menstrual cycles and women with symptomatic fibroids [3]. The SSS (questions 1 to 8) ranges from 8 to 40 (i.e., range $=32$ ). Higher transformed SSS values are indicative of greater symptom severity or bother and lower scores indicate minimal symptom severity. The HRQL has six subscales (i.e., concern, activities, energy/mood, control, self-conscious, and sexual function). Each subscale was created by summing the scores of the items. Higher transformed HRQL score is an indicative of better quality of life. The transformed SSS score and transformed HRQL used in this study analysis were calculated as described by Spies et al. [3].

\section{The safety endpoints}

Adverse events (AEs) were based on patient self-report and collected by recording the self-reported symptoms at site visits and during telephone contacts. AEs including serious adverse events (SAEs) were reviewed by the sponsor and the clinical events committee (CEC). SAEs were defined according to the Society of Interventional Radiology guidelines [33].

\section{Statistical analysis}

The primary analysis tested the null hypothesis, which the proportion of treated women with fibroid shrinkage would be equal to 0.70 , against the alternate hypothesis, which the proportion with fibroid shrinkage would be greater than 0.70 . This hypothesis was evaluated using

Table 1 Patient demographics, number and type of fibroids

\begin{tabular}{|c|c|c|c|c|}
\hline Variable & Category & Peking $(N=58)$ & Xi'an $(N=49)$ & Overall $(N=107)$ \\
\hline Age (years) & Mean (SD) & $45.1(4.50)$ & $43.9(4.50)$ & $44.5(4.50)$ \\
\hline Weight (kg) & Mean (SD) & $62.2(7.60)$ & $58.5(7.80)$ & $60.5(7.90)$ \\
\hline BMI $\left(k g / m^{2}\right)$ & Mean (SD) & $24(2.40)$ & $22.8(2.60)$ & $23.5(2.60)$ \\
\hline \multirow[t]{2}{*}{ Ethnicity } & Asian & 57 (98.3 \%) & 49 (100 \%) & 106 (99.1\%) \\
\hline & Caucasian & $1(1.7 \%)$ & $0(0.0 \%)$ & $1(0.9 \%)$ \\
\hline \multirow[t]{2}{*}{ Number of fibroids } & Total number & 77 & 53 & 130 \\
\hline & Mean (SD) & $1.3(0.50)$ & $1.1(0.30)$ & $1.2(0.50)$ \\
\hline \multirow[t]{3}{*}{ Type of fibroid } & Intramural & $42(54.5 \%)$ & $45(84.9 \%)$ & $87(66.9 \%)$ \\
\hline & Submucosal & $5(6.5 \%)$ & $2(3.8 \%)$ & $7(5.4 \%)$ \\
\hline & Subserosal & 30 (39.0\%) & 6 (11.3\%) & $27.7 \%)$ \\
\hline
\end{tabular}


Table 2 Patients with fibroid shrinkage at 6 months post treatment

\begin{tabular}{lllccc}
\hline Sites & $\begin{array}{l}\text { Evaluable } \\
\text { patients }\end{array}$ & $\begin{array}{l}\text { Number of } \\
\text { successes }\end{array}$ & Proportion & $95 \% \mathrm{Cl}$ & $P$ value \\
\hline Peking & 58 & 52 & 0.90 & $(0.79,0.96)$ & $<.001$ \\
Xi'an & 49 & 47 & 0.96 & $(0.86,1.00)$ & $<.001$ \\
Overall & 107 & 99 & 0.93 & $(0.86,0.97)$ & $<.001$ \\
\hline
\end{tabular}

data for both sites and a two-sided exact-binomial $Z$-test at the 0.05 significance level. Exact Clopper-Pearson $95 \%$ confidence interval for the proportion of patients with fibroid shrinkage at 6 months was calculated.

In addition, the primary analysis was repeated with fibroid shrinkage and was defined more conservatively, that is the proportions of patients with $\geq 10, \geq 20, \geq 30, \geq 40$, and $\geq 50 \%$ reduction in fibroid volume at 6 months was estimated, with the Exact Clopper-Pearson 95 \% confidence interval for the proportions calculated overall and by site. The proportion of patients with fibroid shrinkage 1,3 , and 6 months post treatment with $95 \%$ CI was also calculated. Also, the percent reduction in fibroid volume at 1,3 , and 6 months follow-up were calculated based on fibroid volume measured using MRI. The NPV based on MRI was summarized by site and overall.

The transformed SSS and seven UFS-QoL subscales were summarized for baseline, 1, 3, and 6 months post treatment. The number of observations, mean, standard deviation, and $95 \% \mathrm{CI}$ for the raw values and for change from baseline were presented by site and overall.

Statistical analyses were performed using SAS (ver. 9.3, SAS Institute Inc., Cary, NC 27513, USA).

\section{Results}

\section{Patient demographics}

Three hundred fifty women were screened, and 107 (30.6\%) of them underwent HIFU treatment. A summary of patient screening is shown in Fig. 2. Findings from the MRI screening which includes presence of vertical scar tissue, bowel interference, patients with more than five symptomatic fibroids, fibroids that are too large $\left(>250 \mathrm{~cm}^{3}\right)$ or too deep (skin to fibroid center $>10 \mathrm{~cm}$ ), highly perfused fibroid with excessive vascularity (Funaki type 3),

Table 3 Proportion of patients with reduction in total fibroid volume at 6 months

\begin{tabular}{llll}
\hline $\begin{array}{l}\text { Reduction in total } \\
\text { fibroid volume }(\%)\end{array}$ & Peking $(N=58)$ & Xi'an $(N=49)$ & Overall $(N=107)$ \\
\hline$\geq 10$ & $0.90(0.79,0.96)$ & $0.96(0.86,1.00)$ & $0.93(0.86,0.97)$ \\
$\geq 20$ & $0.89(0.77,0.96)$ & $0.87(0.74,0.95)$ & $0.88(0.80,0.94)$ \\
$\geq 30$ & $0.76(0.62,0.87)$ & $0.74(0.59,0.86)$ & $0.75(0.65,0.83)$ \\
$\geq 40$ & $0.69(0.54,0.80)$ & $0.52(0.37,0.67)$ & $0.61(0.51,0.71)$ \\
$\geq 50$ & $0.46(0.33,0.60)$ & $0.30(0.18,0.46)$ & $0.39(0.29,0.49)$ \\
\hline
\end{tabular}

Table 4 Percent reduction in fibroid volume measured by MRI

\begin{tabular}{lllll}
\hline \multirow{2}{*}{ Site } & \multicolumn{4}{l}{ Months post treatment } \\
\cline { 3 - 5 } Peking & No. patients & 58 & 54 & 6 \\
& Mean (SD) & $20.6(23.00)$ & $43.3(25.10)$ & $51.3(21.60)$ \\
& $95 \% \mathrm{Cl}$ & $(14.6,26.6)$ & $(36.4,50.1)$ & $(45.4,57.2)$ \\
Xi'an & No. patients & 49 & 45 & 46 \\
& Mean (SD) & $20.1(16.60)$ & $39.6(19.40)$ & $48.8(19.00)$ \\
& $95 \% \mathrm{Cl}$ & $(15.3,24.9)$ & $(33.8,45.4)$ & $(43.2,54.5)$ \\
Overall & No. patients & 107 & 99 & 100 \\
& Mean (SD) & $20.4(20.20)$ & $41.6(22.70)$ & $50.2(20.40)$ \\
& $95 \% \mathrm{Cl}$ & $(16.5,24.2)$ & $(37.1,46.1)$ & $(46.1,54.2)$ \\
\hline
\end{tabular}

pedunculated fibroids were the most frequent reason (i.e., $69.5 \%$ ) for a patient not to be enrolled. Also, $29.6 \%$ of the screened women withdrew from study prior to enrollment. All of the 107 treated patients completed the 1-month follow-up visit, 102 (95\%) completed the 3 -month visit, and 100 (93\%) completed the 6-month visit. A summary of patient demographics is presented in Table 1. No MR-HIFU therapies were canceled due to technical failures.

\section{Primary efficacy results \\ Reduction in fibroid volume: patient level analysis}

The overall proportion of women with treatment success (the total fibroid volume of treated fibroids at 6 months plus the error estimate was $90 \%$ or less than the total fibroid volume of treated fibroids at baseline minus the error estimate) was 0.93 (95\% CI 0.86-0.97). The error estimate was based on intra-reader variability calculated by the core laboratory responsible for the image evaluation. The proportion of treatment success is significantly higher than the null hypothesized value of 0.70 for overall and for each site, $p$ value $<0.001$. Treatment success was reported in 52 of the $58(90 \%)$ patients treated at the Peking site and 47 of the 49 (96\%) patients treated in Xi'an Jiaotong site. Table 2 shows the number and proportion of patients with fibroid shrinkage by study site and overall.

\section{Reduction in fibroid volume: fibroid level analysis}

The proportion of patients with $\geq 10, \geq 20, \geq 30, \geq 40$, and $\geq 50 \%$ reduction in fibroid volume 6 months post

Table 5 Summary non-perfused fibroid volume percent (\%NPV) based on MRI evaluation (treatment visit)

\begin{tabular}{lllll}
\hline Variable & Category & Peking $(N=58)$ & Xi'an $(N=49)$ & Overall $(N=107)$ \\
\hline \%NPV & No. patients & 57 & 49 & 106 \\
& Mean (SD) & $55.7(21.10)$ & $53.7(21.30)$ & $54.8(21.20)$ \\
& $95 \% \mathrm{Cl}$ & $(50.1,61.3)$ & $(47.5,59.8)$ & $(50.7,58.8)$
\end{tabular}

\%NPV percent non-perfused volume 


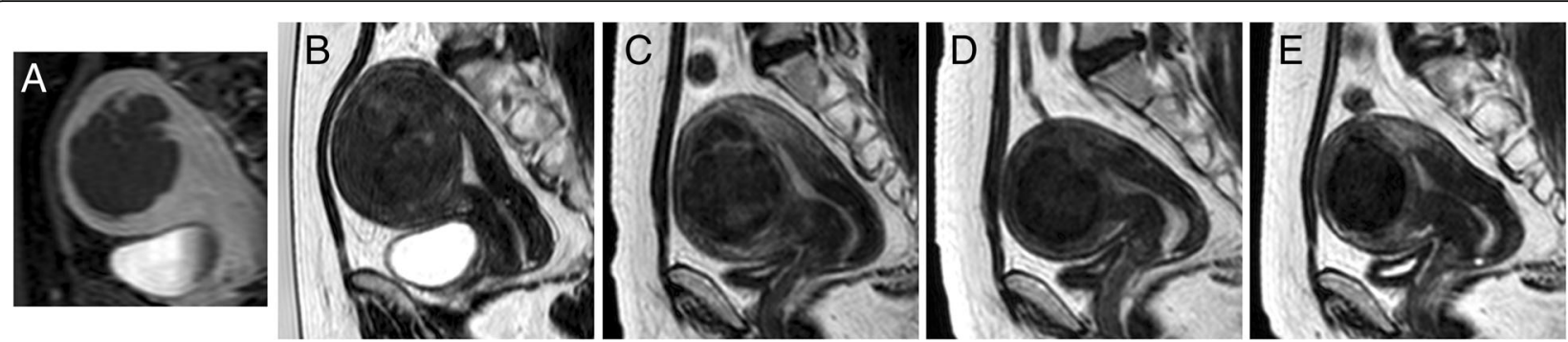

Fig. 3 An example of the progression of fibroid volume reduction after successful treatment: a T1W CE-THRIVE immediately post treatment, b T2W image of the fibroid at treatment day (fibroid volume $144.3 \mathrm{ml}$ ), and c-e T2W images at 1 month (70.3 ml, \% reduction $51.32 \%$ ),

3 months (31.8 $\mathrm{ml}$, \% reduction $74.8 \%$ ), and 6 months $(23.6 \mathrm{ml}$, \% reduction $83.6 \%)$ follow-ups

treatment with the Exact Clopper-Pearson $95 \%$ confidence interval overall and by site are presented in Table 3 . The proportion of patients with at least $40 \%$ reduction in total fibroid volume was 0.61 ( $95 \%$ CI 0.51-0.71).

\section{Secondary efficacy analysis Fibroid-related endpoints}

The percent reduction in fibroid volume at 1,3 , and 6 months follow-up based on fibroid volume measured using MRI both in Peking and Xi'an sites were very consistent between the two sites $(20.6,43.3$, and $51.3 \%$ for Peking site and 20.1, 39.6, and $48.85 \%$ for Xi'an). The number of observations, mean, standard deviation, and $95 \% \mathrm{CI}$ is presented in Table 4. Overall, the mean percent reduction in fibroid volume at 6 months was $50.2 \%$, with the lower bound of the $95 \%$ CI of 46.1. This result indicates that on the average, the fibroid volume reduction for this procedure is significantly greater than $45 \%$. Figure 3 shows an example of the progression of fibroid volume reduction after successful treatment.

Table 5 provides a summary on NPV after treatment. The mean NPV was also very consistent between the two sites (i.e., $55.7 \%$ for the Peking site and $53.7 \%$ for the Xi'an site).

\section{Uterine fibroid symptom quality of life (UFS-QoL)}

The UFS-QoL was assessed at the screening visit and during follow-up at 1, 3, and 6 months. The overall results of UFS-QoL are shown in Table 6. The SSS-transformed score for both sites combined were observed to decline over time. The SSS-transformed score at screening was 34.4 , and 24.0 at 6 months post treatment. The SSS at 6 months is statistically different from the screening SSS at 0.05 level (non-overlapping $95 \% \mathrm{CI}$ ). At the same time, the HRQL-transformed score increased from 73 to 82.2. The HRQL at 6 months is statistically different from the screening HRQL at 0.05 level (non-overlapping $95 \% \mathrm{CI}$ ).

\section{Adverse events}

Table 7 gives an overview of AEs. A total of 366 adverse events were reported, out of which 10 were reported as SAEs. No deaths were reported. A large number of SAEs is explained by four pregnancies, each of which was reported as SAE when they occurred. All pregnant patients terminated their pregnancy voluntarily while enrolled in the trial. Each pregnancy termination was reported as a separate SAE as well. The remaining two SAEs were due to occurrence of breast cancer surgery $(N=1)$ and fibroid-

Table 6 Uterine fibroid symptom severity (UFS-QoL) overall summary

\begin{tabular}{|c|c|c|c|c|c|}
\hline \multirow[b]{2}{*}{ UFS-QoL parameter } & \multirow[b]{2}{*}{ Statistic } & \multirow[b]{2}{*}{$\begin{array}{l}\text { Screening } \\
(N=107)\end{array}$} & \multicolumn{3}{|c|}{ Months post treatment } \\
\hline & & & $\begin{array}{l}1 \\
(N=107)\end{array}$ & $\begin{array}{l}3 \\
(N=102)\end{array}$ & $\begin{array}{l}6 \\
(N=100)\end{array}$ \\
\hline \multicolumn{6}{|l|}{ SSS transformed ${ }^{a}$} \\
\hline & Mean (SD) & $34.4(14.70)$ & $28.1(15.00)$ & $27.2(15.70)$ & $24(16.10)$ \\
\hline & $95 \% \mathrm{Cl}$ & $(31.6,37.2)$ & $(25.2,30.9)$ & $(24.2,30.3)$ & $(20.8,27.2)$ \\
\hline \multicolumn{6}{|l|}{ HRQL transformed ${ }^{a}$} \\
\hline & Mean (SD) & $73(18.80)$ & $78.1(18.00)$ & $78.5(19.50)$ & $82.2(17.30)$ \\
\hline & $95 \% \mathrm{Cl}$ & $(69.4,76.6)$ & $(74.7,81.6)$ & $(74.7,82.4)$ & $(78.7,85.6)$ \\
\hline \multicolumn{6}{|l|}{ Total score } \\
\hline & Mean (SD) & $79.3(25.10)$ & $71.3(24.40)$ & $70.6(26.10)$ & $65.4(24.30)$ \\
\hline & $95 \% \mathrm{Cl}$ & $(74.5,84.1)$ & $(66.7,76)$ & $(65.5,75.7)$ & $(60.5,70.2)$ \\
\hline
\end{tabular}

SSS symptom severity score, HRQL health-related quality of life

${ }^{\mathrm{a}}$ Transformed scores (SSS and HRQL) can range from 0 to 100 . The total score ranges from 37 to a maximum of 185 
Table 7 Summary of adverse events

\begin{tabular}{|c|c|c|c|}
\hline Adverse event & Peking $(N=58)$ & Xi'an $(N=49)$ & Overall $(N=107)$ \\
\hline Total number of adverse events & 224 & 142 & 366 \\
\hline Patients with at least one SAE & $1(1.7 \%)$ & $5(10.2 \%)$ & $6(5.6 \%)$ \\
\hline Total number of serious AEs & $2(0.9 \%)$ & $8(5.7 \%)$ & $10(2.7 \%)$ \\
\hline Pregnancy & $1(1.7 \%)$ & $3(6.1 \%)$ & $4(3.7 \%)$ \\
\hline Termination of pregnancy & $1(1.7 \%)$ & $3(6.1 \%)$ & $4(3.7 \%)$ \\
\hline Ductal carcinoma & $0(0.0 \%)$ & $1(2.0 \%)$ & $1(0.9 \%)$ \\
\hline Fibroid surgery & $0(0.0 \%)$ & $1(2.0 \%)$ & $1(0.9 \%)$ \\
\hline Patients with at least one $\mathrm{AE}$ & 45 (77.6 \%) & $38(77.6 \%)$ & $83(77.6 \%)$ \\
\hline Total number of related AEs & $52(23.2 \%)$ & 107 (74.8 \%) & $159(43.3 \%)$ \\
\hline Abdominal/pelvic pain & $30(51.7 \%)$ & $30(61.2 \%)$ & $60(56.1 \%)$ \\
\hline Skin heating/pain & $25(43.1 \%)$ & $10(20.4 \%)$ & 35 (32.7 \%) \\
\hline Leg pain & 15 (25.9\%) & $5(10.2 \%)$ & $20(18.7 \%)$ \\
\hline Back pain & $11(19.0 \%)$ & $2(4.1 \%)$ & $13(12.1 \%)$ \\
\hline Abdominal distension & $3(5.2 \%)$ & $1(2.0 \%)$ & $4(3.7 \%)$ \\
\hline Buttock pain & $4(6.9 \%)$ & $0(0.0 \%)$ & $4(3.7 \%)$ \\
\hline Leg numbness & $3(5.2 \%)$ & $0(0.0 \%)$ & $3(2.8 \%)$ \\
\hline Skin redness & $1(1.7 \%)$ & $2(4.1 \%)$ & $3(2.8 \%)$ \\
\hline 1st degree skin burn & $1(1.7 \%)$ & $0(0.0 \%)$ & $1(0.9 \%)$ \\
\hline Leg edema/pain & $1(1.7 \%)$ & $0(0.0 \%)$ & 1 (0.9\%) \\
\hline Pubic bone pain & $0(0.0 \%)$ & $1(2.0 \%)$ & $1(0.9 \%)$ \\
\hline Skin edema & $1(1.7 \%)$ & $0(0.0 \%)$ & $1(0.9 \%)$ \\
\hline
\end{tabular}

related minimally invasive surgery $(N=1)$. All SAEs were considered unrelated to study procedure and device.

Out of the non-serious AEs, 159 (43.7 \%) were considered to be related or possibly related to the device. Most patients (i.e., $77.6 \%$ ) reported at least one $\mathrm{AE}$ related to the device, most commonly abdominal/pelvic pain, skin heating/pain, leg pain, and back pain. Of 159 AEs, 153 were recorded during and immediately after HIFU treatment while six of them were $1-3$ days after treatment.

\section{Discussion}

In this study, the effectiveness of MR-HIFU device using a volumetric ablation technique in the treatment of symptomatic uterine fibroids was measured by assessing shrinkage of treated fibroids, which is an objective, quantitative, imaging-based study endpoint.

The study met its pre-defined endpoint, with $93 \%$ (95\% CI 86-97\%) of study patients exhibiting shrinkage of $\geq 10 \%$ fibroid volume shrinkage. In this study, the mean fibroid volume reduction at 6 months was $50.3 \%$ (Table 4). As shown in Table 5, this rate of success was achieved with a mean NPV of $54.8 \%$. NPV as a predictor for fibroid shrinkage and clinical success has been established in one of the latest studies [24]. Given the adequate level of NPV achieved in this study, the fibroid volume is reduced as expected. Note that NPV is expected to further improve as the operators complete their learning curve and gain more experience in patient selection and conducting the procedure [34, 35].

Patients' SSS of the UFS-QoL questionnaire improved after treatment. As shown in Table 6, the mean SSS was reduced from 34 points at baseline to 24 points at 6 months post treatment. A reduction of 10 points or more is considered clinically significant [16]. It must be noted that, in the present population in China, patients scored their own symptoms considerably lower, and their personal health better, than in predicate studies. Patients with similar demographics in Western population typically scored a baseline of over 60 points [24] compared to 34 points in a Chinese population. This difference can most likely be attributed to cultural differences in self-perception. Evidently, a mean SSS of around 30 points was sufficient for the patients to seek treatment for their fibroid symptoms in this study. However, the results of this prospective study demonstrate that the relationship between volume reduction and symptomatic improvement in fibroids following MR-HIFU treatment is not a direct correlation.

In this study, AEs reported were predominantly mild. Ten SAEs were reported, all of which were judged to be unrelated to the treatment procedure. Reported AEs typically relate to heating from the ultrasound beam, and are typically manifested inter-procedurally and resolved 
shortly thereafter. This is in line with previous clinical studies on MR-HIFU treatments of uterine fibroids $[18,23]$. In one patient, buttock/leg/foot pain persisted for 44 days following the HIFU treatment; isolated similar events have been observed previously in studies using other MR-HIFU devices [16, 36]. An independent CEC evaluated the reported adverse events for determination of seriousness and causal relationship with the treatment. The CEC concluded that most of the AEs were minor, and the safety profile of the MR-HIFU system was acceptable.

A limitation of the study was the single-arm design. MR-HIFU treatment was not directly compared to other options for uterine fibroid therapy. Prior experience shows however that randomization can be prohibitive for enrollment when one arm is invasive and the other non-invasive. To limit possible bias, an objective measurement was chosen as primary endpoint for the study, and assessed by an independent CRO core laboratory. The choice of an objective, a quantitative endpoint which can be independently measured, adds strength to the study. It should be noted however that the secondary endpoints provide symptomatic follow-up and present as important a result as the primary image-based endpoint.

\section{Conclusions}

This study demonstrated that MR-HIFU device using a volumetric ablation technique is safe and technically effective and can be utilized in clinically efficient treatments of symptomatic uterine fibroids.

\section{Abbreviations \\ AE: Adverse events; CEC: Clinical events committee; CE-T1W: Contrast-enhanced T1-weighted; HIFU: High-intensity focused ultrasound; MR-HIFU: Magnetic resonance-guided high-intensity focused ultrasound; NPV: Non-perfused volume; PRF: Proton resonance frequency; SAE: Severe adverse events; SSS: Symptom severity score; T2W-TSE: T2-weighted turbo spin echo; UAE: Uterine artery embolization; UFS-QoL: Uterine fibroid symptom quality of life; US: Ultrasound}

\section{Acknowledgements}

The authors thank Tiina Karjalainen (Philips, Vantaa, Finland), Jason Zhang (Philips, Beijing, China), Song Bo (Philips, Beijing, China), and Andy Yu (Philips, Beijing, China) who with patient recruitment, treatment support, data collection, and follow-up during the clinical study sponsored by Philips. All authors approved the final version of the manuscript for submission.

\section{Funding}

This study was an industry-sponsored clinical investigation and funded by Philips.

\section{Availability of data and materials}

Since the trial results are reviewed by CFDA, we unfortunately cannot share the trial's data. The trial data is used for regulatory submission of Philips Sonalleve MR-HIFU in China only.

\section{Authors' contributions}

RC carried out the conceive and design of the study, and participated in drafting the article. BK carried out the conceive and design of the study, acquisition of data, and interpretation of data, and participated in revising it critically for important intellectual content. HB carried out the conceive and design of the study and participated in drafting the article. XH carried out the conceive and design of the study and participated in drafting the article. XW carried out the conceive and design of the study, acquisition of data, analysis and interpretation of data; and participated in drafting the article WB carried out the conceive and design of the study, and participated in drafting the article. YW carried out the conceive and design of the study, and participated in drafting the article. XY carried out the conceive and design of the study, acquisition of data, analysis and interpretation of data, and participated in drafting the article. JY carried out the conceive and design of the study, acquisition of data, analysis and interpretation of data, and participated in drafting the article. JW carried out the conceive and design of the study and acquisition of data, and participated in revising it critically for important intellectual content. MS participated in revising it critically for important intellectual content. AV participated in revising it critically for important intellectual content. QL carried out the conceive and design of the study, and interpretation of data, and participated in drafting the article. All authors read and approved the final manuscript.

\section{Competing interests}

$R C, H B, Y Z, Y W, X H, X Y, X W, J Y, Q L$ declare no personal financial relationships with any organizations that might have an interest in the submitted work in the previous three years and no other relationships or activities that could appear to have influenced the submitted work. BK, JW, MS, and AV are employees of Philips.

\section{Consent for publication}

Not applicable.

\section{Ethics approval and consent to participate}

This study was a multi-center, single arm, non-randomized clinical trial to evaluate the safety and effectiveness of volumetric MR-HIFU system in the treatment of symptomatic uterine fibroid patients in China (sponsored by Philips, clinicaltrials.gov identifier NCT01588899). Local ethics committee approval was obtained from both Clinical Trial Ethic Committee of Peking University First Hospital and The Ethics Review Committees of the First Affiliated Hospital, Medical College, Xi'an Jiaotong University for the protocol prior to study initiation. Written informed consent was obtained from each patient at the screening visit prior to the initiation of any study-related procedures.

\section{Author details}

'Department of Obstetrics \& Gynecology, Beijing Tsinghua Changgung Hospital, Medical Center, Tsinghua University, No. 168 Litang Road, Changping District, Beijing, China. ${ }^{2}$ MR Therapy Division, Philips, Seoul, South Korea. ${ }^{3}$ Department of Obstetrics \& Gynecology, Peking University First Hospital, Beijing, China. ${ }^{4}$ Department of Obstetrics \& Gynecology, First Affiliated Hospital of Xi'an Jiaotong University, Xi'an, China. ${ }^{5}$ Department of Radiology, Peking University First Hospital, Beijing, China. ${ }^{6}$ Department of Radiology, The First Affiliated Hospital of Xi'an Jiaotong University, Xi'an, China. ${ }^{7}$ Philips Research China, Shanghai, China. ${ }^{8}$ MR Therapy Division, Philips, Helsinki, Finland.

Received: 14 June 2016 Accepted: 3 October 2016

Published online: 03 November 2016

\section{References}

1. Stewart EA. Uterine fibroids. Lancet. 2001;357:293-8.

2. Zowall H, Cairns JA, Brewer C, Lamping DL, Gedroyc WM, Regan L. Costeffectiveness of magnetic resonance-guided focused ultrasound surgery for treatment of uterine fibroids. BJOG. 2008;115(5):653-62.

3. Spies JB, Coyne K, Guaou Guaou N, et al. The UFS-QOL, a new diseasespecific symptom and health-related quality of life questionnaire for leiomyomata. Obstet Gynecol. 2002;99(2):290-300.

4. Pritts EA, Parker WH, Olive DL. Fibroids and infertility: an updated systematic review of the evidence. Fertil Steril. 2009;91(4):1215-23.

5. Smart OC, Hindley JT, Regan L, et al. Magnetic resonance guided focused ultrasound surgery of uterine fibroids - the tissue effects of $\mathrm{GnRH}$ agonist pretreatment. Eur J Radiol. 2006;59:163-7.

6. Spies JB, Ascher SA, Roth AR, et al. Uterine artery embolization for leiomyomata. Obstet Gynecol. 2001;98:29-34.

7. Spies JB, Myers ER, Worthington-Kirsch R, et al. The FIBROID Registry: symptom and quality-of-life status 1 year after therapy. Obstet Gynecol. 2005;106:1309-18 
8. Walker WJ, Pelage JP. Uterine artery embolisation for symptomatic fibroids: clinical results in 400 women with imaging follow up. BJOG. 2002;109:1262-72.

9. Cowan B, Sewell P, Howard J, et al. Interventional magnetic resonance imaging cryotherapy of uterine fibroid tumors: preliminary observation. Am J Obstet Gynecol. 2002;186:1183.

10. Sakuhara Y, Shimizu T, Kodama Y, et al. Magnetic resonance-guided percutaneous cryoablation of uterine fibroids: early clinical experiences. Cardiovasc Intervent Radiol. 2006;29:552-8.

11. Whiteman MK, Hillis SD, Jamieson DJ, et al. Inpatient hysterectomy surveillance in the United States, 2000-2004. Am J Obstet Gynecol. 2008;198:34. e1-7.

12. Keshavarz $\mathrm{H}$, Hillis SD, Kieke BA, et al. Hysterectomy surveillance-United States, 1994-1999. MMWR CDC Surveill Summ. 2002;51:1-8.

13. Ishihara $Y$, Calderon $A$, Watanabe $H$, et al. A precise and fast temperature mapping using water proton chemical shift. Magn Reson Med. 1995;34:814-23.

14. Tempany CM, Stewart EA, McDannold N, et al. MR imaging-guided focused ultrasound surgery of uterine leiomyomas: a feasibility study. Radiology. 2003;226(3):897-905

15. Stewart EA, Gedroyc WM, Tempany CM, et al. Focused ultrasound treatment of uterine fibroid tumors: safety and feasibility of a noninvasive thermoablative technique. Am J Obstet Gynecol. 2003;189(1):48-54

16. Hindley J, Gedroyc WM, Regan L, et al. MRI guidance of focused ultrasound therapy of uterine fibroids: early results. AJR Am J Roentgenol. 2004;183:1713-9.

17. Funaki $K$, Fukunishi $H$, Funaki $T$, et al. Mid-term outcome of magnetic resonance-guided focused ultrasound surgery for uterine myomas: from six to twelve months after volume reduction. J Minim Invasive Gynecol. 2007;14:616-21.

18. Stewart EA, Gostout B, Rabinovici J, et al. Sustained relief of leiomyoma symptoms by using focused ultrasound surgery. Obstet Gynecol. 2007;110:279-87.

19. Lenard ZM, McDannold NJ, Fennessy FM, et al. Uterine leiomyomas: MR imaging-guided focused ultrasound surgery-imaging predictors of success. Radiology. 2008;249:187-94.

20. LeBlang SD, Hoctor K, Steinberg FL. Leiomyoma shrinkage after MRI-guided focused ultrasound treatment: report of 80 patients. AJR Am J Roentgenol. 2010;194:274-80

21. Funaki $K$, Fukunishi $H$, Sawada K. Clinical outcomes of magnetic resonanceguided focused ultrasound surgery for uterine myomas: 24-month follow-up. Ultrasound Obstet Gynecol. 2009;34:584-9.

22. Kim YS, Kim JH, Rhim H, et al. Volumetric MR-guided high-intensity focused ultrasound ablation with a one-layer strategy to treat large uterine fibroids: initial clinical outcomes. Radiology. 2012;263(2):600-9.

23. Park MJ, Kim YS, Rhim HC, et al. Safety and Therapeutic efficacy of complete or near-complete ablation of symptomatic uterine fibroid tumors by MR imaging-guided high-intensity focused US therapy. J Vasc Interv Radiol. 2014;25:231-9.

24. Mindjuk I, Trumm CG, Herzog P, et al. MRI predictors of clinical success in MR-guided focused ultrasound (MRgFUS) treatments of uterine fibroids: results from a single centre. Eur Radiol. 2015;25(5):1317-28.

25. Fennessy FM, Tempany CM, McDannold NJ, et al. Uterine leiomyomas: MR imaging-guided focused ultrasound surgery — results of different treatment protocols. Radiology. 2007;243:885-93.

26. Ikink ME, Voogt MJ, Verkooijen HM, et al. Mid-term clinical efficacy of a volumetric magnetic resonance-guided high-intensity focused ultrasound technique for treatment of symptomatic uterine fibroids. Eur Radiol. 2013:23:3054-61.

27. Thiburce AC, Frulio N, Hocquelet A, et al. Magnetic resonance-guided high-intensity focused ultrasound for uterine fibroids: mid-term outcomes of 36 patients treated with the Sonalleve system. Int J Hyperthermia. 2015:31(7):764-70.

28. Machtinger R, Fennessy FM, Stewart EA, et al. MR-guided focused ultrasound (MRgFUS) is effective for the distinct pattern of uterine fibroids seen in African-American women: data from phase III/IV, non-randomized, multicenter clinical trials. J Ther Ultrasound. 2013;1:23.

29. Ticca C, Bignardi T, Zucconi F, et al. Efficacy of MR-guided focused ultrasound surgery (MRgFUS) of uterine fibroids: evaluation of non-perfused volume (NPV), fibroid shrinkage and clinical improvement at 6 months follow-up. Transl Cancer Res. 2014;3(5):413-20.

30. Enholm JK, Köhler MO, Quesson B, et al. Improved volumetric MR-HIFU ablation by robust binary feedback control. IEEE Trans Biomed Eng. 2010;57:103-13.
31. Köhler MO, Mougenot C, Quesson B, Mougenot C, Moonen CT, Sokka SD. Volumetric HIFU ablation under 3D guidance of rapid MRI thermometry. Med Phys. 2009;36:3521-35.

32. Funaki $K$, Fukunishi $H$, Funaki $T$, et al. Magnetic resonance-guided focused ultrasound surgery for uterine fibroids: relationship between the therapeutic effects and signal intensity of preexisting T2 weighted magnetic resonance images. Am J Obstet Gynecol. 2007;196:184. e181-184.e186.

33. Sacks D, McClenny TE, Cardella JF, et al. Society of Interventional Radiology clinical practice guidelines. J Vasc Interv Radiol. 2003;14:S199-202.

34. Rabinovici J, Inbar $Y$, Revel A, et al. Clinical improvement and shrinkage of uterine fibroids after thermal ablation by magnetic resonance-guided focused ultrasound surgery. Ultrasound Obstet Gynecol. 2007;30:771-7.

35. Park MJ, Kim YS, Keserci B, et al. Volumetric MR-guided high-intensity focused ultrasound ablation of uterine fibroids: treatment speed and factors influencing speed. Eur Radiol. 2013:23:943-50.

36. Hesley GK, Felmlee JP, Gebhart JB, et al. Noninvasive treatment of uterine fibroids: early Mayo Clinic experience with magnetic resonance imagingguided focused ultrasound. Mayo Clin Proc. 2006;81(7):936-42.

\section{Submit your next manuscript to BioMed Central and we will help you at every step:}

- We accept pre-submission inquiries

- Our selector tool helps you to find the most relevant journal

- We provide round the clock customer support

- Convenient online submission

- Thorough peer review

- Inclusion in PubMed and all major indexing services

- Maximum visibility for your research

Submit your manuscript at www.biomedcentral.com/submit
) Biomed Central 